\title{
Relaciones familiares desde la perspectiva de adolescentes de Querétaro, México: un estudio a partir del enfoque de la democratización familiar
}

\section{Family relations from the perspective of adolescents from Querétaro, Mexico: a study based on the approach of family democratization}

\author{
Ursula Gayou-Esteva', María-Elena Meza-de-Luna² \\ ${ }^{1}$ IIPSIS Investigación e Intervención Psicosocial, A.C \\ ¿Universidad Autónoma de Querétaro \\ (Rec.: abril de 2017 - Acep.: marzo de 2018)
}

\begin{abstract}
Resumen
Las familias han estado sufriendo múltiples transformaciones en las últimas cinco décadas, experimentando cambios en su estructura, organización y sistema de relaciones. Esta investigación se da a la tarea de hacer una evaluación del grado de democratización con que los adolescentes perciben a sus familias, en el contexto de la ciudad de Querétaro (México). Utilizamos un enfoque cuantitativo con la aplicación de un cuestionario a adolescentes de secundaria ( $n$ = 286, $M=13.01 \pm 1.02$ años) para medir cuatro rasgos de democratización familiar: convivencia, comunicación, violencia y autoritarismo. Encontramos una tendencia en la percepción entre adolescentes hacia evaluar a sus familias con rasgos de democratización. Sin embargo, aún hay rezagos del autoritarismo que se manifiestan en el ejercicio de la violencia como un medio para enfrentar los problemas intrafamiliares, presente en casi la mitad de los hogares estudiados (47.6\%). Los hallazgos son un desafío para académicos/as y profesionales dedicados a la prevención de la violencia, para crear nuevas estrategias capaces de promover la justicia en las relaciones familiares.
\end{abstract}

Palabras clave: Democratización familiar, adolescentes, violencia, Querétaro.

\begin{abstract}
During the last five decades, families have been undergoing multiple transformations, experiencing changes in their structure, organization and relationships. This research evaluates the degree of democratization with which adolescents perceive their families, in the context of the city of Querétaro (Mexico). This research uses a quantitative approach applying a questionnaire to high-school adolescents ( $n=286, M=13.01 \pm 1.02$ years) in order to measure four features of family democratization: family coexistence, communication, violence and authoritarianism. We found a tendency in the perception of adolescents to evaluate their families with traces of democratization. However, there are still traces of authoritarianism that manifest in the use of violence as a means to deal with family problems, which is present in almost half of the households studied (47.6\%). Findings present a challenge to academics and professionals dedicated to prevent violence, so as to create new strategies to promote justice in family coexistence.
\end{abstract}

Keywords: Family democratization, adolescents, violence, Querétaro

Correspondencia: Ursula Gayou Esteva. IIPSIS Investigación e Intervención Psicosocial, A.C. Querétano, México. Correo electrónico: ursulagayou@gmail.com 


\section{Introducción}

Acelerados procesos de cambio político, social e ideológico a nivel macrosocial y el consecuente surgimiento de configuraciones variadas en los hogares, son factores detonantes para que muchas familias actuales estén transitando por nuevos conflictos. Algunas de estas familias atajan dichos conflictos imponiendo el silencio a través de la violencia, mientras que otras, han logrado acceder a soluciones más democráticas. La preponderancia de unas u otras estrategias de afrontamiento de conflictos tiene consecuencias que no se agotan en determinar el nivel de bienestar de las personas que integran dicho grupo familiar, sino que adquieren relevancia a escala social.

Ariza y Oliveira (2001) señalan a la familia como una instancia con capacidad para regular la desigualdad social, bien sea para aminorarla o intensificarla. El ámbito familiar está atravesado por luchas y conflictos que pueden determinar un acceso diferencial a bienes, servicios y recursos simbólicos que promueven la formación de la identidad, de las subjetividades y de las posibilidades de empoderamiento (Giddens, 1991, como se citó en Ariza y Oliveira, 2001, p. 28). En este sentido, el ejercicio del autoritarismo en los hogares, al implicar una distribución desigual de los recursos en función a la adscripción de género y/o generación, es una forma de violencia estructural susceptible de producir consecuencias asociadas a la intensificación de la desigualdad social. Entre dichas consecuencias, Ariza y Oliveira (2001) destacan el embarazo adolescente, la desnutrición, la deserción escolar, las adicciones y en un lugar protagónico, la violencia familiar. Esto último debido a que la violencia familiar tiene un fuerte impacto en la calidad de vida y la salud de las personas (Secretaría de Salud, 2006).

Uno de los retos en el desarrollo de estrategias de prevención de la violencia en la familia, es contar con diagnósticos que permitan comprender mejor la situación del grupo y de la comunidad donde se pretende hacer intervención. En este sentido y con el propósito de coadyuvar en el desarrollo de una sociedad más justa, esta investigación tiene como objetivo evaluar el grado de democratización con que los y las adolescentes perciben a sus familias; considerando cuatro rasgos: convivencia, comunicación, violencia y autoritarismo.

La democracia entendida como igualdad en el acceso a derechos y responsabilidades, es una figura propia de lo macrosocial que debe construirse y sostenerse también desde lo microsocial (Marín y Uribe, 2017). Esta es la idea central del enfoque de la democratización familiar que retomamos para este estudio. Enlazando los constructos de la teoría de género y la perspectiva de Derechos Humanos, la mirada de la democratización de las familias "permite revisar, cuestionar y resignificar los discursos hegemónicos sobre familia e infancia, relaciones de poder y género, concepciones de autoridad y discursos y prácticas patriarcales, que aún continúan ancladas a las prácticas cotidianas de las familias" (Marín y Uribe, 2017, p. 32).

El contexto social actual comporta múltiples presiones para las familias y fomenta cambios importantes tanto en las estructuras que estas asumen, como en las pautas de relación entre sus miembros. Para las personas más jóvenes, quienes transitan por etapas cruciales de desarrollo físico y psicológico, las relaciones armónicas o conflictivas en sus familias adquieren especial relevancia y son determinantes no sólo de su bienestar presente, sino también de aprendizajes, vivencias y decisiones que incidirán en sus vidas a futuro. Frente a ello, se hace pertinente el estudio sobre cómo perciben los/as adolescentes las relaciones al interior de sus hogares, como una manera de entender mejor la situación de este grupo etario y tener elementos para el desarrollo de intervenciones en beneficio de su calidad de vida.

Internacionalmente se ha documentado una alta incidencia de exposición a la violencia en población de adolescentes, entre $29.4 \%$ y $50 \%$ experimentan violencia en sus hogares (Garrido, Culhane, Petrenko y Taussig, 2011; McDonald, Jouriles, Ramisetty-Mikler, Caetano y Green, 2006). Sin embargo, en México, la reciente Encuesta Nacional de Niños, Niñas y Mujeres [ENIM] 2015 revela una problemática aún más extendida: $63.1 \%$ de los niños, niñas y adolescentes respondieron -por cuenta propia o con apoyo de sus tutores/as- que los métodos de disciplina empleados en sus hogares incluyen alguna forma de violencia, mientras que $43.7 \%$ han padecido algún episodio de violencia física (Instituto Nacional de Salud Pública [INSP] y UNICEF México, 2016). Esta información está en concordancia con la obtenida en la Encuesta Nacional de Identidad y Valores 2015, en la que $61.6 \%$ de las personas mayores de 15 años está total o parcialmente de acuerdo frente a la pregunta: "¿se debe permitir o no se debe permitir a los padres que peguen a los niños para corregirlos?" (Flores, 2015).

Crecer en contextos violentos tiene graves consecuencias para las personas. Los estudios de Buckner, Beardslee y Bassuk (2004) refieren que la exposición a la violencia incrementa las posibilidades de padecer depresión, ansiedad, síntomas traumáticos, estrés crónico y sentimientos de inseguridad. También se ha señalado que la violencia vivida en edades tempranas conlleva afectaciones multidimensionales que involucran aspectos psicológicos, de desarrollo y de socialización (Bolívar, Convers y Moreno, 2014). De ahí la necesidad de evaluar las relaciones familiares y la violencia en la familia, desde la perspectiva de la población más joven.

Hasta el momento, las investigaciones en torno a la violencia familiar se han centrado más en documentar su impacto en las personas y en las relaciones familiares, que en estudiar la prevalencia de factores relacionales estructurales que permitan desarrollar caminos de resolución temprana frente a este complejo problema (Bolívar et al., 2014). Asimismo, a pesar de que en países como México está vigente el papel protagónico de la familia como el núcleo de socialización más valorado por las personas (Flores, 2015), pocos estudios aprecian las relaciones familiares multifactorialmente para aproximar perspectivas acerca del bienestar de sus integrantes. Por ello, en la presente investigación nos enfocamos en caracterizar las relaciones familiares de una muestra de adolescentes reuniendo indicadores que permitan entrever áreas de oportunidad revisadas a la luz del enfoque de la democratización familiar.

\section{Familia y hogar: conceptos y tendencias}

La palabra "familia" tiene un extendido uso coloquial. Comúnmente se da por hecho su sentido apelando a referentes compartidos y modelos difusos, lo cual conlleva el riesgo de atribuirle un sentido universal y facilita pasar por alto la complejidad que entraña definir este término desde las ciencias sociales. Las definiciones de familia se enfrentan con dos riesgos: ser tan amplias que en ellas quepa cualquier grupo hu- 
mano o ser tan acotadas que la realidad de las familias supere la conceptualización. Para sortear estos riesgos, se puede elegir una conceptualización abstracta de familia, capaz de alojar la diversidad de sus configuraciones. En términos de Palacios (2009), desde una perspectiva sociológica la familia puede ser entendida como:

Una institución social que se organiza a partir de relaciones de parentesco, las cuales están normadas por pautas y prácticas sociales ya establecidas y que además, contiene interacciones que pueden implicar la co-residencia o rebasar los límites de la unidad residencial (...) es una institución que moldea la personalidad, la reproducción social y las relaciones sociales (p. 189)

Por otro lado, cuando se quiere hacer referencia al grupo de personas que cohabitan en una casa, considerando que estas pueden tener o no tener lazos de parentesco, se emplea el término hogar. De acuerdo con Palacios (2009), el hogar es una forma de organización social entre individuos que comparten una residencia y realizan "de manera conjunta, en armonía o en conflicto, las diversas actividades que favorecen el mantenimiento, reposición y reproducción de la vida humana" (pp.189-190).

\section{Hogares y familias actuales: transiciones y diversidad}

En los últimos cincuenta años se han registrado importantes diversificaciones en la estructura de los hogares, respecto del modelo tradicional compuesto por una pareja heterosexual unida en primeras nupcias y su descendencia (Flores, 2015). Estos cambios en los hogares se relacionan con procesos sociales macroestructurales que inciden en los estilos de vida de la comunidad y con el paso del tiempo, se reflejan en tendencias demográficas. La globalización de un sistema capitalista neoliberal o de libre mercado ha erosionado la función benefactora del Estado, dando lugar a severas crisis económicas que afectan a las familias y con frecuencia les impulsan a apoyarse en las labores de cuidado de niñas/os o personas dependientes, conformando hogares denominados "extensos" donde además de la familia nuclear (biparental o monoparental), habita al menos otro/a pariente que no es hijo/a soltero/a (Echarri, 2009). También es posible identificar la gran influencia ideológica y política que han alcanzado los movimientos feministas en su lucha por la reivindicación de los derechos de las mujeres, impulsando la aparición nuevas imágenes de la feminidad menos centradas en la maternidad y con ello, la construcción de nuevos modelos de familia: por ejemplo, familias con jefatura femenina, familias sin hijas/os o con un número reducido de estas/os (Ariza y Oliveira, 2001; Flores, 2015).

No obstante, el hecho de que los hogares se hayan diversificado a nivel estructural, no necesariamente se traduce en que dichos hogares asuman nuevas pautas de organización o de convivencia. Los cambios no ocurren de manera lineal, ni son asumidos sin resistencia por quienes los enfrentan, pues trastocan paradigmas en lo concerniente a los roles de género y a las relaciones entre mujeres y hombres, tanto intergenéricas como intragenéricas (Flores, 2015; Marín y Uribe, 2017). En distintos niveles, los valores emergentes relacionados con la igualdad de género, el manejo de conflictos y el respeto a los derechos humanos, confluyen con valores tradicionales asociados con sistemas de autoridad rígidos y patriarcales.

\section{Patriarcado de ayer y hoy}

El concepto patriarcado nombra al "conjunto de relaciones sociales entre los hombres que tiene una base material y que, si bien son jerárquicas, establecen o crean una interdependencia y solidaridad entre los hombres que les permiten dominar a las mujeres" (Hartmann, como se citó en Amorós, 2008, p. 40). Es decir, los varones están subordinados los unos a los otros en razón de factores étnicos y de clase social, sin embargo, "esa subordinación es de naturaleza tal que no llega a impedir que los varones, en su conjunto, dominen al conjunto de las mujeres" (p. 40).

En las sociedades tradicionales, el patriarcado se erigió con base en una clara división sexual del trabajo que destinaba a los varones a realizar labores asociadas con la producción de bienes y servicios, mientras que las mujeres eran responsables de las labores necesarias para garantizar la reproducción. La lógica patriarcal asignó superioridad y poder a los varones y a sus roles productivos, sobre las mujeres y sus roles reproductivos. Asimismo, se sexualizaron los espacios de la vida: lo público y sus instituciones representantes devinieron ámbitos masculinos, y lo privado (territorio del hogar y hogar de los afectos) se feminizó. En función de las transformaciones históricas en los modos de producción y las formas de gobernabilidad, refiere Fernández (1993), la subordinación femenina: Ha cambiado sus figuras económicas, sociales, políticas y subjetivas, así como los argumentos religiosos, legales y científicos por los que ha querido justificarse (...) pero sin que se suprimieran la desigualdad ni las formas visibles e invisibles de sus violentamientos (pp. 120-121).

\section{Poder y autoridad en las familias}

La distribución de las relaciones de poder y de autoridad en las familias está ligada al sistema patriarcal. Aunque las mujeres han ido ocupando nuevos espacios sociales y subjetivos de dignificación y resistencia (Fernández, 1993; Galeana y Vargas, 2015), la organización patriarcal subsiste debido a que los roles que atribuye a mujeres y a varones, diferenciados entre sí y jerárquicos, son naturalizados por las actoras y los actores sociales frente a la fuerza de la costumbre . Fernández (1993) destaca la necesidad de detenerse a considerar que las nuevas prácticas de género no han superado a las viejas, sino que coexisten con ellas y generalmente en tensión conflictiva: "la adquisición de nuevos espacios de inserción no ha liberado a las mujeres de casi ninguna de sus responsabilidades en sus espacios tradicionales" (p. 135). De igual manera, menciona que formas de violencia menos visibles que el maltrato físico (pero no menos eficaces), "se ponen en práctica en la familia cotidianamente a través de la desigualdad en la distribución del dinero, del poder, de las responsabilidades domésticas, de las opciones de realización personal, etc." (Fernández, 1993, p. 118).

La convivencia en el hogar, siempre está regulada por sistemas de autoridad subyacentes. La autoridad puede emanar del ejercicio de un poder coercitivo (autoridad-autoritaria) o bien, del ejercicio de un poder legítimo ligado a la posesión de habilidades valoradas (autoridad-autorizada), tal como plantea León (2003). De acuerdo con Schmukler y Alonso (2009), los sistemas de autoridad son mecanismos que legitiman jerarquías entre hombres y mujeres y fijan contratos implícitos sobre sus funciones con base en determinadas creencias y 
valores sociales acerca del género y de la misma autoridad. Tanto en México como en el resto del mundo aún prevalecen valores patriarcales, por lo que la autoridad máxima en las familias generalmente se le reconoce a un varón, quien a su vez se asume como jefe de familia (Galeana y Vargas, 2015; Schmukler y Alonso, 2009). La primacía de un sistema de autoridad que jerarquiza a los individuos de acuerdo con su sexo biológico está en el origen del autoritarismo pues, como señalan Galeana y Vargas (2015) "los atavismos patriarcales suponen que el hombre debe mandar y la mujer obedecer" (p. 147). Consecuentemente, es posible advertir que en la medida en que los hogares asumen sistemas de autoridad más legítimos y flexibles, así como prácticas más democráticas, se convierten en semilleros de ciudadanas y ciudadanos: "porque entonces hablamos de hombres y mujeres que crecen con valores que los impulsan a participar socialmente, que se apropian de sus derechos y, por lo tanto, no conciben una cultura autoritaria que los invisibilice" (Schmukler y Alonso, 2009, p. 55).

\section{Rasgos autoritarios y democráticos en las familias}

Aunque en las familias generalmente se observa la confluencia de ciertas prácticas autoritarias con otras más bien democráticas (Marín y Uribe, 2017; Rojas, 2016), a nivel teórico se suele caracterizar a las familias como autoritarias o democráticas en función de cuál estilo predomina en el ejercicio del poder y la toma de decisiones al interior de estas. Las denominadas "familias autoritarias" se caracterizan porque las normas de convivencia que rigen las relaciones entre sus integrantes no son negociadas sino impuestas, muchas veces a través de formas explícitas de violencia. Se trata de familias en las que no se da lugar a un ejercicio de diálogo ni de escucha de las necesidades de cada quien (Schmukler y Alonso, 2009). En el otro lado de un continuo con muchos matices, se observa que en la actualidad algunas familias regulan su relaciones y reparten sus roles a través de prácticas más democráticas, que constituyen "un escenario de enlace intergeneracional propuesto en sentido de derechos humanos y una con-vivencia orientada por la ética de la reciprocidad, la responsabilidad entre todos los integrantes (...) y la convivencia que aporte a la resolución de los conflictos" (Marín y Uribe, 2017, pp. 44-45). De acuerdo con Schmukler y Alonso (2009), las familias que se rigen por un sistema democrático reúnen las siguientes características: (a) simetría de poderes en las relaciones de pareja, (b) consulta entre las autoridades y los miembros del grupo para la toma de decisiones, (c) división de responsabilidades y deberes entre las autoridades, (d) coordinación, comunicación, apoyo y respeto entre quienes ejercen la autoridad, (e) aceptación de la diferencia de autoridad y de las disparidades entre cada una de las personas que la ejercen.

\section{La influencia del autoritarismo familiar en los y las adolescentes}

La adolescencia es aquella etapa del desarrollo en la que se afianzan los bastiones de la identidad de las personas (Kaufman, como se citó en Secretaría de Salud, 2006). En la adolescencia, las experiencias de la infancia comienzan a resignificarse con base en las vivencias más actuales y asumen configuraciones más duraderas y estables. El contexto en el que se desenvuelven las y los adolescentes es determinante para facilitar o bien, obstaculizar el desarrollo de valores democráticos, habilidades relacionales y de capacidades asociadas al desempeño académico y/o laboral. De acuerdo con
Schmukler y Alonso (2009), un contexto familiar autoritario donde se establecen jerarquías basadas en el género de sus integrantes y se censuran los conflictos a través de la violencia, constituye un escenario adverso para el óptimo desarrollo de las personas. Al reproducir estereotipos de género, este tipo de contexto dificultará a las mujeres adolescentes la adquisición de autonomía, mermando su capacidad para tomar decisiones y establecer límites que las protejan frente a posibles abusos de poder. A su vez, favorecerá la legitimación del uso de violencia de parte de los varones, naturalizando la subordinación de las mujeres y de las generaciones más jóvenes.

En el tránsito por la adolescencia, muchos varones y muchas mujeres toman decisiones que conllevan fuertes repercusiones para el resto de sus vidas. Estudiar, elegir una profesión y/o una pareja, ingresar al mundo laboral e iniciar una vida sexual, constituyen algunas de estas decisiones cruciales. Todas estas elecciones son influidas por factores sociales, económicos, étnicos, de clase y de género, pero uno de los factores en mayor medida determinantes es el contexto familiar (Luna, Laca y Mejía, 2011), de modo que en las elecciones adolescentes se refleja lo hablado en familia, pero también lo silenciado; la autonomía desarrollada, pero también la autonomía negada; los derechos asumidos y los derechos denegados; las relaciones democráticas y las violentas; los sistemas de autoridad legítimos y los autoritarismos; el afecto y la indiferencia. Así, una familia autoritaria puede ser la antesala de complejos problemas sociales y de salud para los y las adolescentes.

\section{Método}

Para alcanzar el objetivo de investigación utilizamos un enfoque cuantitativo con la aplicación de una encuesta en una secundaria general del Estado de Querétaro. A continuación, se detalla el diseño de la investigación.

\section{Participantes}

Realizamos un muestreo aleatorio por conglomerados (correspondiendo a grupos). Los criterios de inclusión para la participación fue tener entre 11 y 17 años y ser estudiante de la escuela secundaria donde se realizó el estudio.

Participaron estudiantes de secundaria $(n=286)$ entre los 11 y 16 años ( $M=13.01 \pm 1.02$ años) de las cuales $54.7 \%$ eran mujeres y $45.3 \%$ eran hombres. Siendo que la escuela tiene ocho grupos de primer grado, cuatro de segundo y cuatro de tercero, hicimos una selección aleatoria ponderada. La muestra por grado académico quedó conformada con el $53.1 \%$ de alumnado del primer año, $26.2 \%$ de segundo y $20.6 \%$ de tercero. Las personas participantes señalaron que viven con la madre (95.1\%), el padre (79.4\%), hermanos/as (91.3\%), abuelas (23.1\%), abuelos (15.0\%) y otras personas (29.4\%; e.g., padrastro, tías/os). La mayoría de los y las adolescentes tienen uno (35.3\%) o dos (36.4\%) hermanos/as. Solo el 3.7\% de las personas participantes tienen 5 hermanos/as o más. La muestra de adolescentes consideró que su situación económica era muy buena $13.3 \%$, buena $50.2 \%$, regular $32.6 \%$, mala $3.5 \%$ y muy mala $0.4 \%$. 


\section{Instrumentos}

Desarrollamos un instrumento de propósito específico para atender los objetivos de una investigación más amplia que indagó datos censales, el tipo de estructura y ciclo de vida de la familia, aspectos para evaluar el grado de democratización familiar, violencia sexual y estereotipos de género. En este comunicado nos abocaremos a los rasgos de democratización familiar. Evaluamos la percepción de adolescentes sobre su familia en cuatro aspectos: la convivencia (i.e., comparto momentos de convivencia agradable con personas de mi familia), la comunicación (i.e., platico con personas de mi familia sobre lo que me pasa o lo que me preocupa), manejo del conflicto con violencia (i.e., ocurren peleas en las que se humilla, insulta o golpea) y el autoritarismo en la toma de decisiones (i.e., mi papá o mi mamá, toman decisiones sin tomar en cuenta la opinión de los/as demás). En esta escala hicimos un análisis de componentes principales con el método Varimax, donde encontramos dos dimensiones: una de familias democráticas que incluye los reactivos de comunicación y convivencia, y la segunda sub-escala es de familias autoritarias compuesta de los ítems violencia y autoritarismo. La validación del instrumento se realizó con entrevistas cognitivas $(n=3)$ con adolescentes de entre 12 y 13 años.

\section{Procedimiento}

Presentamos el diseño de la investigación al director de la escuela, con quien acordamos el procedimiento para su ejecución, fijando una calendarización de actividades y acordando la disposición de los recursos necesarios. Los instrumentos de recolección de datos fueron evaluados y avalados por la dirección de la secundaria.

Una de las primeras acciones en la institución fue el proceso de validación del instrumento, para lo cual procedimos a realizar entrevistas cognitivas (Desimone y Le Floch, 2004; García, 2011), con la intención de detectar problemas que la población de adolescentes podía tener para comprender las instrucciones del cuestionario, de las preguntas formuladas y de sus opciones de respuesta. Al mismo tiempo, se validaba si las preguntas eran entendidas en mismo sentido con el que fueron creadas. Se acordaron los días y horarios para aplicar dichas entrevistas a 3 estudiantes ( 1 hombre y 2 mujeres) que cursaban primer y segundo grado.de los cuales se entrevistó 2 de primero y 1 de segundo. Tras realizar las entrevistas cognitivas se hicieron ajustes al instrumento que luego se aplicó en una prueba piloto. Finalmente, aplicamos el instrumento a los grupos seleccionados aleatoriamente. El instrumento se contestó en aproximadamente 30 minutos, y se aplicó entre el 18 y el 25 de octubre de 2013.

El presente trabajo de investigación se apegó a las pautas éticas estipuladas por la American Psychological Association (APA, 2010); se ofrecieron consentimientos informados y se respetó en todo momento la confidencialidad y el anonimato de las personas voluntarias que participaron.

\section{Análisis de datos}

El análisis de datos lo hicimos con el SPSS (2003), usamos la estadística descriptiva, y realizamos análisis de componentes principales con pruebas de esfericidad. También realizamos prueba de medias t de student, al 95\% de confianza para com- parar las edades de hombres y mujeres participantes. Para hacer el estudio comparativo de los rasgos de democratización familiar entre los grupos de hombres y mujeres, hicimos pruebas de independencia Chi-cuadrada de Pearson.

\section{Resultados}

La mayoría de las y los adolescentes consideran tener una buena relación con su familia. El $88.6 \%$ señaló que comparten momentos agradables con su familia al menos algunas veces y $11.4 \%$ señaló que rara vez o nunca lo hacen (ver Figura 1). Así, la gran mayoría evalúa tener en lo general una buena convivencia con su familia. Sin embargo, cuando evaluamos cuestiones puntuales como el estilo de comunicación abierto, los indicadores son menos contundentes. Específicamente, más de la mitad (67.2\%) considera que puede llegar a platicar con sus familias sobre lo que les pasa, mientras que $32.8 \%$ indicó que esto sucede rara vez o nunca (ver Figura 2).

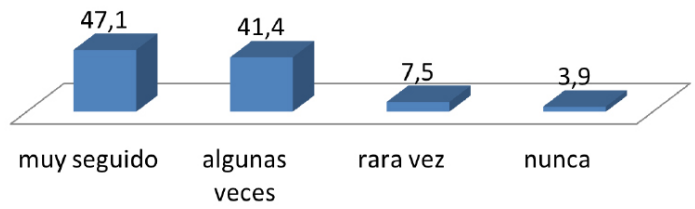

Figura 1. Percepción de adolescentes sobre la convivencia familiar

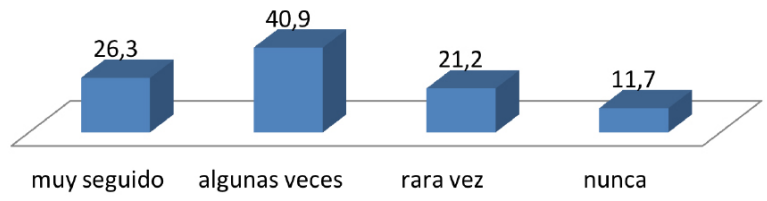

Figura 2. Percepción de adolescentes sobre la comunicación familiar

En cuanto a las percepciones sobre actos violentos tales como humillaciones, insultos y golpes, encontramos que casi la mitad $(47.6 \%)$ de los y las adolescentes han enfrentado algún conflicto familiar con violencia. Aunque solamente el $2.9 \%$ señaló que les ocurre de manera sistemática (ver Figura 3). 


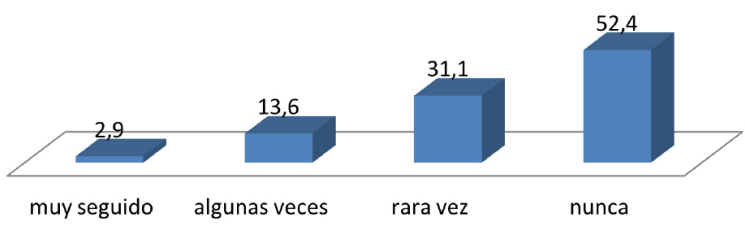

Figura 3. Percepción de adolescentes sobre peleas en la familia

Con respecto a la percepción del autoritarismo de las madres y los padres en el entorno familiar, encontramos que si bien una parte importante de las y los jóvenes (57.2\%) perciben que ha habido algún autoritarismo de parte del padre o de la madre, sólo el $21.2 \%$ considera que sucede con relativa frecuencia y $78.8 \%$ lo percibe como poco frecuente o inexistente. Es decir, la mayoría de los y las estudiantes evalúan a sus familias con rasgos democráticos en tanto que se toman decisiones consensuadas.

La Figura 5 muestra los resultados en conjunto de los dos rasgos democratizadores, donde se puede apreciar que los ítems de la dimensión de autoritarismo (i.e., violencia y autoritarismo) tienen una relación de Pearson positiva que, aunque es significativa, no se presenta con gran potencia $(r=0.25, p<$ 0.01).

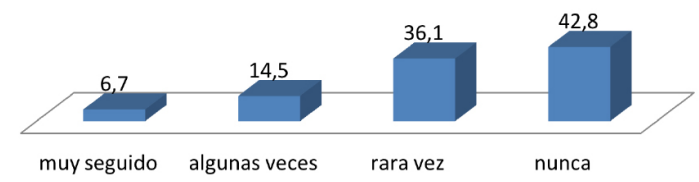

Figura 4. Percepción de adolescentes sobre el autoritarismo familiar

\section{Comparación entre hombres y mujeres}

Encontramos que no hay una diferencia estadísticamente significativa entre la edad de hombres y de mujeres $(t=-.0721$, $p=0.47$ ). En cuanto a los rasgos de democratización familiar, tampoco hallamos alguna diferencia estadísticamente significativa entre los géneros en ninguno de los cuatro indicadores [entre corchetes comparación de rasgos democratizadores altos de mujeres vs. hombres]: convivencia $\chi 2(3, N=286)=1.72$, $p=0.63 ;[90 \%$ vs. 90\%], comunicación $\chi 2(3, N=286)=7.13$, $p=0.07 ;[70 \%$ vs. $60 \%]$, violencia $\chi 2(3, N=286)=3.35, p=$ $0.34 ;[10 \%$ vs. $10 \%]$, autoritarismo $\chi 2(3, N=286)=2.66, p=$ $0.45 ;$ [ $10 \%$ vs. $20 \%]$.

\begin{tabular}{|c|c|c|c|c|c|}
\hline \multirow{6}{*}{ 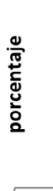 } & \multirow{6}{*}{$\begin{array}{r}60,0 \\
50,0 \\
40,0 \\
30,0 \\
20,0 \\
10,0 \\
, 0\end{array}$} & \multirow{2}{*}{\multicolumn{4}{|c|}{ 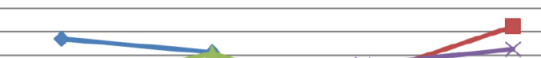 }} \\
\hline & & & & & \\
\hline & & \multirow{2}{*}{\multicolumn{3}{|c|}{$F$}} & \\
\hline & & & & & \\
\hline & & \multicolumn{2}{|c|}{$\%$} & \multicolumn{2}{|c|}{$\Longrightarrow$} \\
\hline & & muy seguido & algunas veces & rara vez & nunca \\
\hline \multicolumn{2}{|c|}{$\begin{array}{l}\text { Comparto momentos de } \\
\text { convivencia agradable con } \\
\text { personas de mi familia }\end{array}$} & 47,1 & 41,4 & 7,5 & 3,9 \\
\hline \multicolumn{2}{|c|}{$\begin{array}{c}- \text { Ocurren peleas en las que se } \\
\text { humilla, insulta o golpea }\end{array}$} & 2,9 & 13,6 & 31,1 & 52,4 \\
\hline \multicolumn{2}{|c|}{$\begin{array}{c}\text { Platico con personas de mi } \\
\text { familia sobre lo que me pasa o } \\
\text { lo que me preocupa }\end{array}$} & 26,3 & 40,9 & 21,2 & 11,7 \\
\hline \multicolumn{2}{|c|}{$\begin{array}{l}\text { Mi papá o mi mamá, toman } \\
\text { decisiones sin tomar en cuenta } \\
\text { la opinión de los(as) demás }\end{array}$} & 6,7 & 14,5 & 36,1 & 42,8 \\
\hline
\end{tabular}

Figura 5. Comparación sobre la percepción en adolescentes sobre rasgos de democratización familiar

\section{Discusión}

Si bien una significativa mayoría de las y los adolescentes comparten momentos de convivencia agradable con sus familias con relativa frecuencia (88.6\%), una tercera parte de la muestra (32.8\%) refirió que rara vez o nunca platica con sus familias sobre lo que les pasa. Una posible explicación para que la comunicación en el núcleo familiar tenga un resultado menos contundente que la convivencia intrafamiliar, es que en esta etapa de vida las personas tienden a desarrollar una alta identificación con su grupo de pares (Tarrant, MacKenzie y Hewitt, 2006) especialmente en la adolescencia temprana (Brown, Eicher y Petrie, 1986), por lo que la comunicación tiende a ser más frecuente con su grupo etario. No obstante, investigaciones recientes han encontrado que las personas jóvenes que refieren tener poca comunicación con sus padres y madres, tienen una probabilidad $30 \%$ mayor de presentar ideación suicida (Pérez-Amezcua, et al., 2010). En función de esta y otras correlaciones antes señaladas entre los rasgos de autoritarismo en las familias y los problemas en la juventud, se recomienda que en futuras investigaciones se incluyan mayor cantidad de reactivos para evaluar conductas concretas sobre democratización familiar y profundicen en las cualidades de estos hallazgos.

Respecto a las respuestas sobre actos violentos, el que casi la mitad de la muestra (47.6\%) haya experimentado alguna forma de violencia en su familia es un problema preocupante que actualiza la necesidad de desarrollar iniciativas de intervención que favorezcan el surgimiento de alternativas de diálogo y manejo de conflictos desde una perspectiva democrática. Sin desmedro de lo anterior, los eventos de violencia familiar parecen estar menos extendidos entre la población del presente estudio, respecto de los resultados que reportan otras investigaciones contemporáneas en la materia, con población mexicana en rangos de edad más amplios. La Encuesta Nacional de Género 2014 registró que 6 de cada 10 personas mayores de 15 años en el país padecieron violencia física en el seno familiar (Galeana y Vargas, 2015). Una tasa muy similar de niños, niñas y adolescentes (63.1\%) ha sufrido violencia de diversos tipos incorporada en los métodos de disciplina que emplean en sus hogares, de acuerdo con la ENIM 2015 (INSP y UNICEF México, 2016). Incorporando la posibilidad de que los resultados obtenidos a partir de este estudio reflejen avances en términos de la eliminación de la violencia en las familias con 
hijos/as adolescentes, será necesario conducir nuevas investigaciones que confirmen esta interpretación.

En cuanto a la percepción sobre el ejercicio de autoritarismos en las familias, la mayoría (78.8\%) de los y las estudiantes ubicó que son poco frecuentes o nulos, por lo que parece predominar un estilo democrático en la toma de decisiones familiares. Si bien, estos hallazgos son un indicativo de que podría estar en marcha un proceso de transformación familiar hacia la democratización, futuros estudios podrán ahondar en las características de dicho proceso y con qué prácticas se concreta para las y los adolescentes, dado que es probable que la toma de decisiones aún no alcance a este sector de la población.

Aunque con base en el presente estudio es posible ubicar una tendencia hacia la democratización en las familias, aún hay rezagos del autoritarismo que se manifiestan en el ejercicio de la violencia como un medio de enfrentar los problemas y en la falta de consideración de las opiniones en familia para la toma de decisiones. Estos dos aspectos son un reto para especialistas de la academia y profesionales en prevención de la violencia, que convocan a generar estrategias de intervención acordes con las nuevas realidades de las familias.

El bienestar y el desarrollo de la sociedad mexicana no van ser procurados por el Estado y sus instituciones representantes a corto o mediano plazo. La violencia estructural y los altos índices de pobreza en nuestro país ofrecen un panorama desalentador para quienes soñamos con la justicia social. Muchos caminos que apuntan hacia este horizonte negado desde la política, están siendo inaugurados desde la sociedad civil. Creemos que si las familias acceden a prácticas democráticas, el futuro colectivo también se transforma. El presente trabajo nos confirma que se están gestando cambios con miras a la democratización, y nos invita a diseñar acciones más específicas para sostener y potenciar tales cambios.

\section{Referencias}

American Psychological Association. (2010). Publication manual of the American Psychological Association (6a ed.). Washington, DC: Author.

Amorós, C. (2008). Mujeres e imaginarios de la globalización, Reflexiones para una agenda teórica global del feminismo. Santa Fe: Homo Sapiens.

Ariza, M. y Oliveira, O. (2001). Familias en transición y marcos conceptuales en redefinición. Papeles de Población, 7(28), 9-39. Recuperado de http://www. redalyc.org/articulo.oa?id $=11202802$

Bolívar, L., Convers, A. y Moreno, J. (2014). Factores de riesgo psicosocial asociados al maltrato infantil. Psychologia Avances de La Disciplina, 8(1), 6776. Recuperado de http://www.redalyc.org/ pdf/2972/297231283007.pdf

Brown, B., Eicher, S. y Petrie, S. (1986). The importance of peer group ("crowd") affiliation in adolescence. Journal of Adolescence, 9, 73-96. doi:10.1016/S01401971(86)80029-X

Buckner, J., Beardslee, W. y Bassuk, E. (2004). Exposure to Violence and Low-Income Children's Mental Health: Direct, Moderated, and Mediated Relations. American Journal of Orthopsychiatry, 74(4), 413-423. doi:10.1037/0002-9432.74.4.413
Desimone, L., Le Floch, K. C. (2004). Are We Asking the Right Questions? Using Cognitive Interviews to Improve Surveys in Education Research. Educational Evaluation and Policy Analysis, 26(1), 1-22. doi:10.3102/01623737026001001

Echarri, C. (2009). Estructura y composición de los hogares en la Endifam. En C. Rabell (Ed.), Tramas familiares en el México contemporáneo. Una perspectiva sociodemográfica (pp.143-175). Distrito Federal: Universidad Autónoma de México/Instituto de Investigaciones Sociales/El Colegio de México.

Fernández, A. (1993). La mujer de la ilusión. Pactos y contratos entre hombres y mujeres. Buenos Aires: Paidós.

Flores, J. (2015). Sentimientos y resentimientos de la nación. Distrito Federal: Universidad Autónoma de México/Instituto de Investigaciones Jurídicas.

Galeana, P. y Vargas, P. (2015). Géneros asimétricos. Representaciones y percepciones del imaginario colectivo. Distrito Federal: Universidad Autónoma de México/Instituto de Investigaciones Jurídicas.

García, A. (2011). Cognitive interviews to test and refine questionnaires. Public Health Nursing, 28(5), 444-450. doi:10.1111/j.1525-1446.2010.00938.x

Garrido, E., Culhane, S., Petrenko, C. y Taussig, H. (2011). Psychosocial Consequences of Intimate Partner Violence (IPV) Exposure in Maltreated Adolescents: Assessing More than IPV Occurrence. Journal of Family Violence, 26(7), 511-518. doi:10.1007/ s10896-011-9386-0

González, R. (2012, octubre. Diferentes diferencias: el transfeminismo como un reto frente a la interseccionalidad. Ponencia presentada en el Segundo Encuentro de Reflexiones Cuir, realizado en la Universidad Autónoma de Querétaro, Querétaro, México..

Instituto Nacional de Salud Pública y UNICEF México. (2016). Encuesta Nacional de Niños, Niñas y Mujeres 2015 - Encuesta de Indicadores Múltiples por Conglomerados 2015, Resultados Principales. Ciudad de México: Autor. Recuperado de https://www.insp.mx/ enim2015/informe-resultados-2015.html

León, M. (2003). Sistema de género, autoridad en relación conyugal. Autoridad femenina, autoridad masculina. En M. León (Ed.), La representación social del trabajo doméstico. Un problema en la construcción de la identidad femenina (pp. 75-84). México: Benemérita Universidad Autónoma de Puebla.

Luna, A., Laca, F. y Mejía, J. (2011). Bienestar subjetivo y satisfacción con la vida de familia en adolescentes mexicanos de bachillerato. Psicología Iberoamericana, 19(2), 17-26. Recuperado de http://www.redalyc.org/articulo.oa?id=133921440003

Marín, A. y Uribe, J. (2017). El cuidado y la crianza como mediadores en la democratización de las relaciones familiares. Revista de Trabajo Social e Intervención Social, 1(23), 23-50. doi:10.25100/prts.v0i23.4584

McDonald, R., Jouriles, E., Ramisetty-Mikler, S., Caetano, R. y Green, C. (2006). Estimating the number of American children living in partner-violent families. Journal of Family Psychology, 20(1), 137-142. doi:10.1037/0893-3200.20.1.137

Palacios, P. (2009). Hogar, género y prácticas alimentarias. En P. Palacios (Coord.), Una visión polisémica de la mujer en Querétaro (pp. 175-221). Querétaro: Plaza y Valdes Editores. 
Pérez-Amezcua, B., Rivera-Rivera, L., Atienzo, E., De Castro, F., Leyva-López, A. y Chávez-Ayala, R. (2010). Prevalencia y factores asociados a la ideación e intento suicida en adolescentes de educación media superior de la República mexicana. Salud Pública de México, 52(4). doi:10.1590/S003636342010000400008

Rojas, O. (2016). Mujeres, hombres y vida familiar en México. Persistencia de la inequidad de género anclada en la desigualdad social. Revista Interdisciplinaria de Estudios de Género, 2(3), 73-101. doi:10.24201/ eg.v2i3.4

Schmukler, B. y Alonso, X. (2009). Bases conceptuales y teóricas para una convivencia democrática en la familia. En B. Schmukler y X. Alonso (Coords.), Democratización familiar en México: experiencias de un proyecto de prevención de violencia familiar (pp. 25-61). Distrito Federal: Instituto de Investigaciones Dr. José María Luis Mora.

Secretaría de Salud. (2006). Informe Nacional sobre Violencia y Salud. Distrito Federal: Secretaría de Salud.

Tarrant, M., MacKenzie, L. y Hewitt, L. (2006). Friendship group identification, multidimensional self-concept, and experience of developmental tasks in adolescence. Journal of Adolescence, 29(4), 627640. doi:10.1016/j.adolescence.2005.08.012 